\title{
Highly oriented $\mathrm{SrTiO}_{3}$ thin film on graphene
}

\section{substrate}

Sang A Lee ${ }^{\dagger, \ddagger, \perp}$, Jae-Yeol Hwang ${ }^{\S, \perp}$, Eun Sung Kim ${ }^{\S}$, Sung Wng Kim ${ }^{*}, \|$, and Woo Seok

Choi $^{*}+$

${ }^{\dagger}$ Department of Physics, Sungkyunkwan University, Suwon, 16419, Korea

${ }^{\ddagger}$ Institute of Basic Science, Sungkyunkwan University, Suwon, 16419, Korea

${ }^{\S}$ Center for Integrated Nanostructure Physics, Institute for Basic Science (IBS), Suwon 16419, Korea

"Department of Energy Sciences, Sungkyunkwan University, Suwon 16419, Korea

KEYWORDS: $\mathrm{SrTiO}_{3}$, graphene, thin film, pulsed laser deposition, interface, epitaxy, diffusion barrier, $\mathrm{Si}$

ABSTRACT: Growth of perovskite oxide thin films on Si in crystalline form has long been a critical obstacle for the integration of multifunctional oxides into Si-based technologies. In this study, we propose pulsed laser deposition of a crystalline $\mathrm{SrTiO}_{3}$ thin film on a $\mathrm{Si}$ using graphene substrate. The $\mathrm{SrTiO}_{3}$ thin film on graphene has a highly $(00 l)$-oriented crystalline structure which results from the partial epitaxy. Moreover, graphene promotes a sharp 
interface by highly suppressing the chemical intermixing. The important role of graphene as a 2D substrate and diffusion barrier allows expansion of device applications based on functional complex oxides.

Functional perovskite oxides exhibit a variety of emergent physical phenomena such as superconductivity, 2D electron liquid behavior, multiferroicity, catalytic activity, and thermoelectricity, and have high potential for application to opto-electronic and energy devices. ${ }^{1-5}$ One of the main obstacles for the practical utilization of such functionalities of perovskite oxides is their compatibility with the existing Si-based technology. In other words, high-quality single-crystalline epitaxial perovskite oxide thin films and heterostructures are grown almost exclusively on single-crystalline perovskite substrates, e.g., $\mathrm{SrTiO}_{3}$, owing to the structural and chemical affinities of the perovskite building blocks.

One of the overarching goals for enabling the practical application of functional oxides is, therefore, to grow single-crystalline $\mathrm{SrTiO}_{3}$ thin films on $\mathrm{Si}^{6-12}$ The $\mathrm{SrTiO}_{3}$ layer serves as a universal template for the growth of most perovskites, and thus, various functional oxide systems might become readily compatible with Si-based electronics. However, the growth of single-crystalline $\mathrm{SrTiO}_{3}$ thin films on $\mathrm{Si}$ is not trivial, since most conventional growth procedures on a Si substrate result in a polycrystalline or an amorphous phase of $\mathrm{SrTiO}_{3}$. The difficulty in the thin film growth arises mainly from the thermal mismatch between Si and oxides and the chemical stability of Si in their vicinity. ${ }^{11}$ Even though the lattice mismatch (a compressive strain of $\sim 1.7 \%$ ) between $\mathrm{SrTiO}_{3}$ and $\mathrm{Si}$ is rather small, ${ }^{10}$ there is a significant discrepancy in their thermal expansion coefficients. More importantly, Si becomes 
chemically unstable over $400^{\circ} \mathrm{C}$, resulting in the formation of a detrimental intermediate layer such as amorphous $\mathrm{SiO}_{2}\left(a-\mathrm{SiO}_{2}\right)$ or silicides $\left(\mathrm{SrSi}_{2}\right.$ and/or $\left.\mathrm{TiSi}_{2}\right)$ at the oxide/Si interface. ${ }^{7,} 11$, ${ }^{13-15}$ In an attempt to minimize the influence of the interface and enhance the crystallinity of the $\mathrm{SrTiO}_{3}$ thin film, previous studies employed complicated approaches such as stepped growth, ultraviolet ozone treatment, and the use of high-flux sources. ${ }^{7,11-12,16}$ Most of these examples were based on molecular beam epitaxy (MBE) which was quite successful in obtaining single-crystalline $\mathrm{SrTiO}_{3}$. Nevertheless, the use of more accessible growth techniques such as sputtering and pulsed laser deposition (PLD) can also be envisaged. While PLD has some drawbacks such as limitations in the sample size, the growth of singlecrystalline $\mathrm{SrTiO}_{3}$ thin films on Si could economically expand the study of complex oxide materials systems on $\mathrm{Si}$.

Recently, graphene has been recognized as a 2D layer that facilitates the growth of crystalline layers. ${ }^{17-19}$ Graphene is a 2D monolayer of carbon atoms in a honeycomb lattice structure, and it promotes van der Waals epitaxy to materials such as GaAs and other 2D layered materials. ${ }^{17}$ Due to this weak van der Waals interaction instead of typical strong covalent or ionic bonding, discrepancies in the lattice constant and crystalline symmetry between the thin film and graphene substrate are much less prominent than those in a film deposited on conventional substrates such as $\mathrm{SiO}_{2} / \mathrm{Si}^{20}$ Moreover, graphene is chemically and thermally stable, because of which it serves as an ideal substrate for epitaxial and/or single-crystalline thin film growth. Finally, given the advantage of graphene to be transferred to any arbitrary substances, including amorphous $\mathrm{SiO}_{2}$ and glass, graphene substrate can facilitate the realization of ubiquitous applications of high-quality single-crystal materials. Such advantageous characteristics of graphene as a 2D substrate allow us to explore the growth of single-crystalline perovskite oxides on graphene. 
In this paper, we report the growth of a highly oriented $\mathrm{SrTiO}_{3}$ thin film on $\mathrm{Si}$ by using graphene as the 2D substrate. Despite the large lattice and symmetry mismatches, the $\mathrm{SrTiO}_{3}$ thin film shows a highly $(00 l)$-oriented crystal structure on the graphene transferred onto $\mathrm{SiO}_{2} / \mathrm{Si}$ substrate. In addition, the graphene layer promotes a sharp interface without the presence of a detrimental intermediate layer, which suggests that the graphene also serves as a suitable diffusion barrier.

In order to examine the suitability of graphene as a 2D substrate for crystalline complex oxides, we used PLD to grow $\mathrm{SrTiO}_{3}$ thin films on graphene-covered $a-\mathrm{SiO}_{2} / \mathrm{Si}$ substrates. The chemical-vapor-deposition-grown graphene was transferred onto $a-\mathrm{SiO}_{2} / \mathrm{Si}(001)$ substrate. PLD was subsequently used to grow $\mathrm{SrTiO}_{3}$ thin films on graphene substrate at $700^{\circ} \mathrm{C}$. Low oxygen partial pressure $\left(\sim 10^{-6}\right.$ Torr $)$ was employed in order to prevent oxidization of the graphene layer. For comparison, $\mathrm{SrTiO}_{3}$ thin films were also deposited on a bare $a-\mathrm{SiO}_{2} / \mathrm{Si}$ substrate at the same time.

Structural characterization of the $\mathrm{SrTiO}_{3}$ thin film on graphene substrate confirmed a crystalline structure with predominant $(00 l)$ orientation, as shown schematically in Figure 1a. Figure $1 \mathrm{~b}$ shows x-ray diffraction (XRD) $\theta-2 \theta$ scans and x-ray reflectivity (XRR) results of the $\mathrm{SrTiO}_{3}$ thin film grown on the $a-\mathrm{SiO}_{2} / \mathrm{Si}$ substrate with (red) and without (blue) graphene. The XRD result of the $\mathrm{SrTiO}_{3}$ thin film on graphene shows clear (00l) $\mathrm{SrTiO}_{3}$ peaks, indicating a highly (00l)-oriented thin film. While weak signatures of (110), (111), and (211) $\mathrm{SrTiO}_{3}$ peaks (at $2 \theta=32.50^{\circ}, 40.03^{\circ}$, and $57.84^{\circ}$, respectively) are also present indicating that the film is not fully single-crystalline, there is a stark distinction between the $\mathrm{SrTiO}_{3}$ thin film on graphene and $a-\mathrm{SiO}_{2} / \mathrm{Si}$. Indeed, the thin film on $a-\mathrm{SiO}_{2} / \mathrm{Si}$ without graphene does not suggest any crystalline structures, except for miniscule hints of (110), (111), (002), and (211) $\mathrm{SrTiO}_{3}$ peaks. Instead, the wide bump at low $2 \theta$ angles indicates that, as predicted, the film is 
largely amorphous on Si. The XRD $\phi$-scan shown in the inset of Figure $1 \mathrm{~b}$ further supports the local ordering of $\mathrm{SrTiO}_{3}$ thin film with four-fold symmetry on graphene substrate. The XRR results also reveal a strong influence of the graphene layer on the growth of the $\mathrm{SrTiO}_{3}$ thin film. Clear interference fringes are observed for $\mathrm{SrTiO}_{3}$ on graphene at grazing incident angles indicating a well-defined $\mathrm{SrTiO}_{3}$-graphene interface and the $\mathrm{SrTiO}_{3}$ surface compared to the sample without the graphene layer, most probably due to the substantially reduced intermixing at the interface.

Transmission electron microscopy (TEM) further confirms the highly oriented crystalline nature of the $\mathrm{SrTiO}_{3}$ thin film on graphene substrate. The cross-sectional TEM image of the $\mathrm{SrTiO}_{3}$ thin film with the graphene layer (Figure 2a) shows $(00 l)$-oriented columnar texture with the local epitaxial growth of $\mathrm{SrTiO}_{3}$, which is in good agreement with the XRD results. The electron diffraction pattern for the well-ordered region (orange box, Figure 2c) exhibits clear spots in the reciprocal lattice, indicating a single-crystalline feature with $[00 l]$ orientation. For another region, twin structures are observed (blue box, Figure 2d). Nevertheless, all the grains are oriented along the $[00 l]$ direction. The high-resolution image in Figure 2e with the intensity line profile of the A- and B-site cations confirms the formation of the expected perovskite structure. In contrast, in the case of the $\mathrm{SrTiO}_{3}$ thin film grown directly on $a-\mathrm{SiO}_{2} / \mathrm{Si}$, atomic orderings are observed only partially, indicating an amorphous phase. We further analyzed magnified TEM images near the interface, as shown in Figures $2 \mathrm{f}$ and $2 \mathrm{~g}$. The $\mathrm{SrTiO}_{3}$ thin film on graphene substrate shows clear interface and well-arranged atomic structure. The termination layer can be roughly estimated as $\mathrm{TiO}_{2}$, which coincides with our expectation as discussed below. On the other hand, the $\mathrm{SrTiO}_{3}$ thin film without graphene shows largely ambiguous interface. 
The coherent growth of the $\mathrm{SrTiO}_{3}$ thin film on graphene might seem rather surprising at first sight, especially considering the mismatch in crystalline symmetry between the film and substrate. In particular, the (111) surface of $\mathrm{SrTiO}_{3}$ seems to be epitaxially more favorable for being attached on top of the honeycomb structure of graphene, due to its hexagonal symmetry. However, this is not the case apparently, because of the following reasons. First, the lattice mismatch for epitaxial growth is actually greater for (111)-oriented $\mathrm{SrTiO}_{3}$ than for (001)oriented $\mathrm{SrTiO}_{3}$ on graphene. A lattice mismatch calculation reveals that the epitaxial strain induced by graphene on the (111) $\mathrm{SrTiO}_{3}$ thin film is $\sim 11.23 \%$, whereas it reduces to $8.72 \%$ (armchair direction) and $-5.40 \%$ (zigzag direction) for the (001) $\mathrm{SrTiO}_{3}$ thin film if we consider a simple square box on top of the hexagonal network (see Figure S1 in Supporting Information). Second, the surface energy $\left(E^{\mathrm{S}}\right)$ is lowest for the $(001)$ surface of $\mathrm{SrTiO}_{3}$. According to a recent density functional theory calculation, $E^{\mathrm{S}}(100)\left(\approx 6.8 \mathrm{eV} / \mathrm{nm}^{2}\right)$ is the lowest among the surface energies of low Miller-index surfaces, by a factor of at least three. ${ }^{21}$ When ablated species arrive at heated substrates, atoms tend to form a new surface with the lowest surface energy. Third, an atomic termination layer for the (001) $\mathrm{SrTiO}_{3}$ surface $\left(\mathrm{TiO}_{2}\right.$ layer) has higher adsorption and migration energies on graphene compared to that for the (111) surface (Ti-only layer). In particular, oxygen $(4.79 \mathrm{eV})$ has higher adsorption energy than does $\mathrm{Ti}(3.27 \mathrm{eV})$, which suggests that it is more favorable to form a $\mathrm{TiO}_{2}$ layer on top of graphene instead of a Ti-only layer. On the other hand, Sr has the lowest adsorption energy $(0.33 \mathrm{eV})$ among the elements, suggesting that the Ti-containing layer should be attached on graphene. ${ }^{22}$ This is in agreement with our TEM observation.

The discussion on the bonding nature and epitaxial strain provides further insight into the growth of the highly oriented $\mathrm{SrTiO}_{3}$ thin film on graphene substrate. We first demonstrate that the bonding between graphene and $\mathrm{SrTiO}_{3}$ is not purely a van der Waals interaction. The 
adsorption energy discussed above can promote a stronger bonding nature between the carbon and Ti/oxygen ions, which can induce an epitaxial strain. While the detailed nature of the chemical bonding is beyond the scope of the present study, the evidence of the epitaxial strain can be found from the structural characterizations. The out-of-plane lattice constant of the $\mathrm{SrTiO}_{3}$ layer calculated from the XRD $(00 l)$ peaks is $\sim 3.896 \AA$ (Figure $\left.1 \mathrm{~b}\right)$. This value is in close agreement with the TEM results shown in Figure 2e. On the other hand, the in-plane lattice constant of the $\mathrm{SrTiO}_{3}$ thin film is $\sim 3.923 \AA$, from an off-axis XRD measurement. The bulk lattice constant of single-crystal $\mathrm{SrTiO}_{3}$ is $3.905 \AA$, indicating a tensile-strained $\mathrm{SrTiO}_{3}$ thin film on graphene. ${ }^{23}$ It should further be noted that the lattice constant calculated from XRD peaks other than the main $(00 l)$ peaks is almost consistent with the bulk value. This indicates that the highly oriented main part of the $\mathrm{SrTiO}_{3}$ thin film is epitaxially strained, whereas other small disoriented regions might be partially relaxed. The moderate strength of the bonding between 2D graphene and the $\mathrm{SrTiO}_{3}$ thin film seems to promote the highly oriented crystalline growth by overcoming the symmetry and lattice mismatches. ${ }^{17}$ Another possible mechanism of the partial epitaxial growth notwithstanding with the previous discussion is thermal mismatch between $\mathrm{SrTiO}_{3}$ and graphene. ${ }^{24}$ The thermal expansion coefficients of $\mathrm{SrTiO}_{3}$, graphene, and $\mathrm{Si}$ are $9.0 \times 10^{-6} \mathrm{~K}^{-1},-8.0 \times 10^{-6} \mathrm{~K}^{-1}$ and $2.5 \times 10^{-6}$ $\mathrm{K}^{-1}$, respectively, exhibiting a large difference.

In addition to facilitating the partially epitaxial growth of $\mathrm{SrTiO}_{3}$, the graphene layer efficiently limits the intermixing of elements during the highly energetic PLD. While such an effect has been demonstrated within perovskite oxide heterostructures, ${ }^{25}$ the effect shown in the present study is much more pronounced owing to the structural robustness of graphene. As shown in Figure 2a, the $\mathrm{SrTiO}_{3}$ thin film grown on graphene substrate has a sharp interface, which is also confirmed from the XRR result (Figure 1b). In contrast, for the thin 
film without graphene, it is rather difficult to identify the interface between the $\mathrm{SrTiO}_{3}$ thin film and the $a-\mathrm{SiO}_{2} / \mathrm{Si}$ substrate, as shown in Figure 2b. This indicates severe elemental intermixing at the interface during the high temperature deposition. Furthermore, the obscure interface adversely influences the lattice arrangement of the entire thin film and results in the formation of an amorphous phase of the $\mathrm{SrTiO}_{3}$ layers. The observation of a well-defined interface in the $\mathrm{SrTiO}_{3}$ thin film on graphene substrate should be also beneficial for controlling the physical properties, since the possible $\mathrm{SiO}_{x}$ layer is subject to direct tunneling leakage and poor reliability. ${ }^{26}$ This result indicates that the graphene layer plays the role of a robust diffusion barrier without requiring complicated approaches for the stabilization of the heterointerface. The use of graphene substrate within PLD is comparable to the Sr layer engineering in MBE, which also reduces the intermixing. ${ }^{27}$

Figure 3 shows the Raman spectrum of the $\mathrm{SrTiO}_{3}$ grown on graphene. The spectra of the $\mathrm{SrTiO}_{3}$ thin film without graphene and that of pristine graphene transferred onto a $\mathrm{Si}$ substrate are also shown for comparison. First, we observe the Raman peaks originating from the graphene layer for the $\mathrm{SrTiO}_{3}$ on graphene, although both $\mathrm{G}\left(\sim 1600 \mathrm{~cm}^{-1}\right)$ and $2 \mathrm{D}(\sim 2680$ $\left.\mathrm{cm}^{-1}\right)$ peaks are rather broad. In addition, we notice D peak $\left(\sim 1360 \mathrm{~cm}^{-1}\right)$, which can be possibly attributed to the disorder induced by the growth of the $\mathrm{SrTiO}_{3}$ thin film at high temperature. The 2D peak for the $\mathrm{SrTiO}_{3}$ on graphene shows a slight blue-shift compared to the bare graphene. This shift might be due to a combined effect of strain and oxidation in graphene layer. ${ }^{28}$ Three peaks originating from the $\mathrm{SrTiO}_{3}$ thin film (indicated by yellow arrows) are also visible: $\mathrm{TO}_{1}\left(\sim 100 \mathrm{~cm}^{-1}\right), \mathrm{LO}_{4}\left(\sim 800 \mathrm{~cm}^{-1}\right)$, and an unidentified peak at $\sim 1360 \mathrm{~cm}^{-1}$. $^{29}$ The peak at $\sim 1360 \mathrm{~cm}^{-1}$, which overlaps with the D peak of graphene, has been previously reported for the $\mathrm{SrTiO}_{3}$ thin film, although it has not been attributed to any specific phonon mode. ${ }^{30}$ The presence of the Raman peaks and the enhancement of the $\mathrm{LO}_{4}$ 
peak originating from the $\mathrm{SrTiO}_{3}$ thin film on graphene substrate in comparison to that from the $\mathrm{SrTiO}_{3}$ thin film without graphene suggest the successful formation of the $\mathrm{SrTiO}_{3}$ layer on top of graphene substrate.

In summary, pulsed laser deposition of a highly-orientated $\mathrm{SrTiO}_{3}$ thin film with a sharp interface on Si was demonstrated via the introduction of graphene as a 2D substrate and a diffusion barrier. The moderate strength of the bonding between the carbon and $\mathrm{SrTiO}_{3}$ layers facilitated the growth of the predominantly $(00 l)$-oriented $\mathrm{SrTiO}_{3}$ thin film on graphene substrate. The bonding between graphene and the $\mathrm{SrTiO}_{3}$ thin film induced the partial epitaxial growth generating an in-plane tensile strain, which was evidenced from various structural analyses. Given the extraordinary structural features of our pulsed laser deposited $\mathrm{SrTiO}_{3}$ thin film on graphene substrate, we expect better integration of functional transition metal oxides into Si-based technology. 


\section{FIGURES}
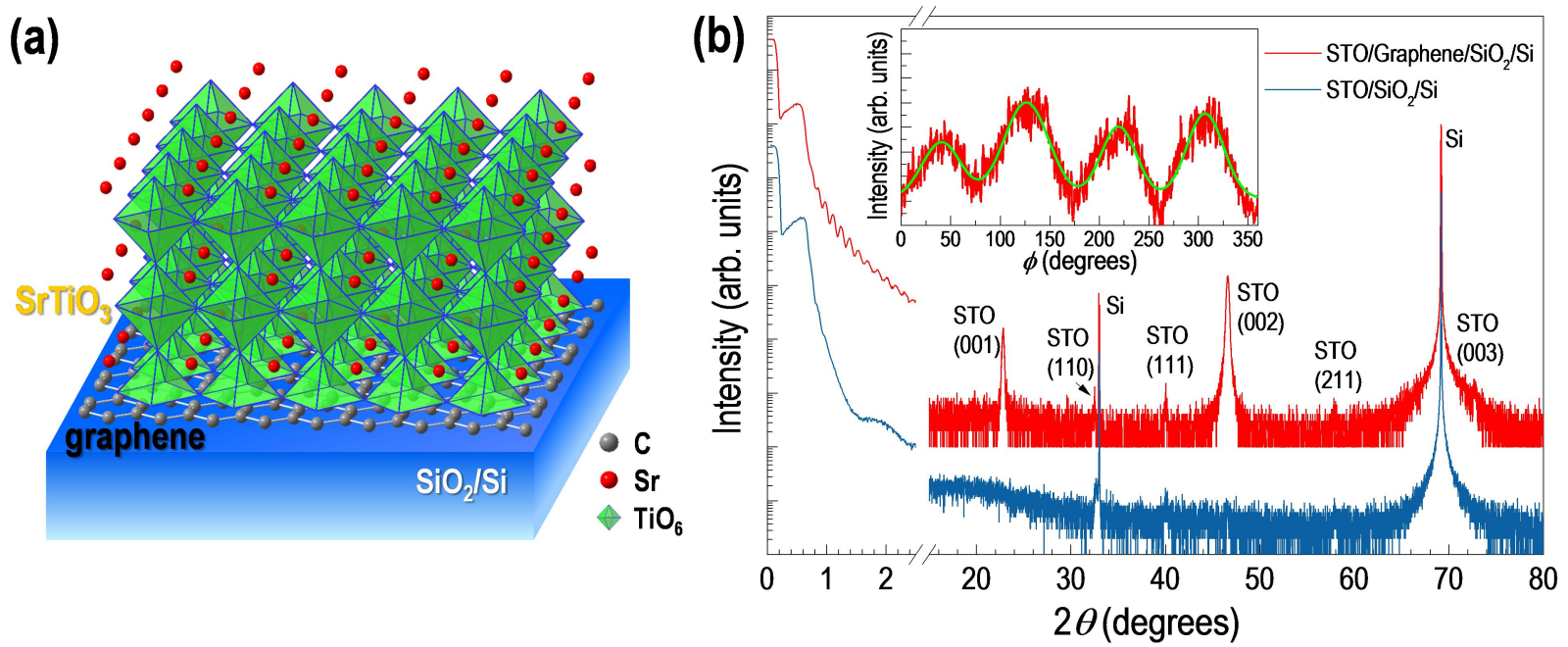

Figure 1. Growth of highly oriented $\mathrm{SrTiO}_{3}$ thin film on 2D graphene substrate. (a) A schematic representation of the $(00 l)$-oriented perovskite $\mathrm{SrTiO}_{3}$ thin film on graphene substrate. (b) X-ray diffraction results of $\mathrm{SrTiO}_{3}$ thin films grown on $a-\mathrm{SiO}_{2} / \mathrm{Si}$ substrates with and without graphene show a substantial difference in crystal structures. For the film on graphene (red curve), strong (00l) Bragg reflection peaks of $\mathrm{SrTiO}_{3}$ are clearly observed, indicating the highly oriented $\mathrm{SrTiO}_{3}$ thin film. On the other hand, the film without graphene does not show any significant diffraction peak indicating a largely amorphous structure. The inset shows XRD $\phi$-scan result of $\mathrm{SrTiO}_{3}$ thin film on graphene substrate, for the 103 reflection. 

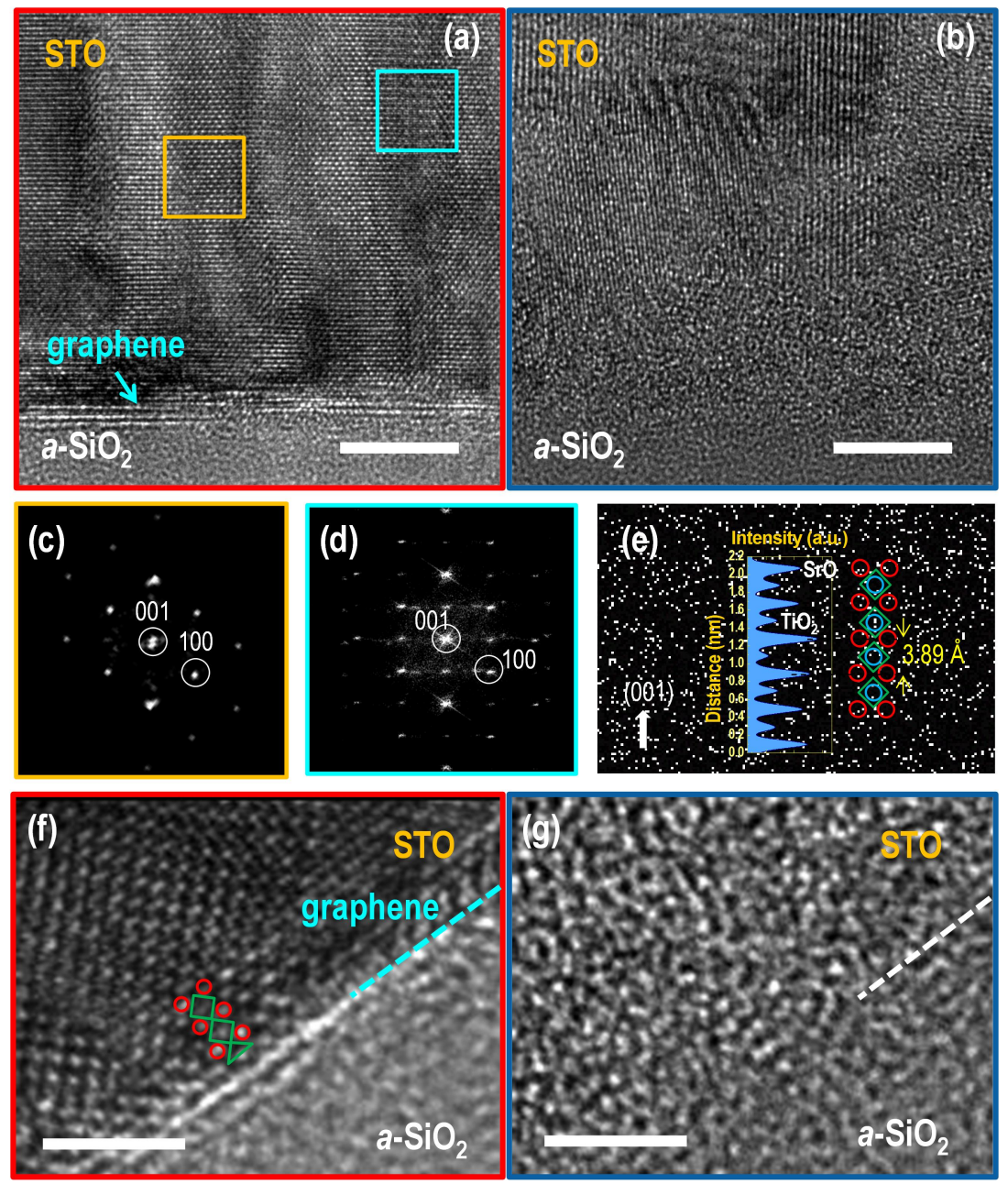

Figure 2. Transmission electron microscopy images of $\mathrm{SrTiO}_{3}$ thin films with and without graphene. The $\mathrm{SrTiO}_{3}$ thin films (a) with and (b) without the graphene show crystalline and amorphous structures, respectively, which is consistent with the XRD results. The film with graphene shows a well-defined interface owing to the structural integrity of graphene. The electron diffraction patterns in the (c) orange and (d) blue boxes show single-crystalline and twin structures, respectively, within the images of the $\mathrm{SrTiO}_{3}$ thin film on graphene substrate. (e) Magnified image of the single-crystalline region. The line profile shows that the $c$-axis lattice parameter is $3.89 \AA$, which is in good agreement with the XRD results. Magnified images of $\mathrm{SrTiO}_{3}$ thin films (f) with and (g) without graphene around the interface. The scale bars represent $5 \mathrm{~nm}$ ( $\mathrm{a}$ and $\mathrm{b}$ ) and $2 \mathrm{~nm}$ (f and g), respectively. 


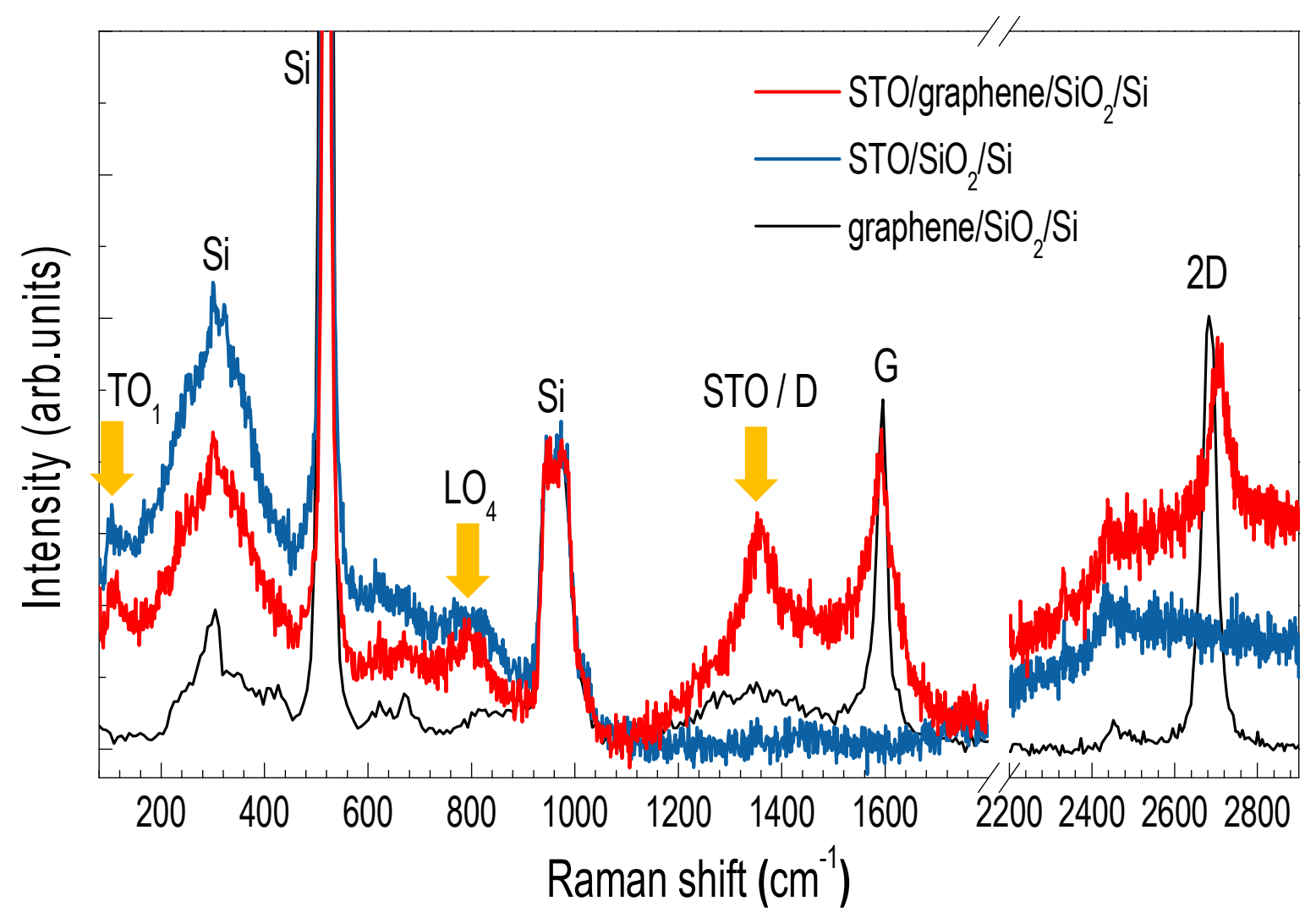

Figure 3. Phonon spectra analyses of $\mathrm{SrTiO}_{3}$ thin films on graphene. The Raman spectra of the $\mathrm{SrTiO}_{3}$ thin films on the $a-\mathrm{SiO}_{2} / \mathrm{Si}$ substrate with (red) and without (blue) graphene show strong $\mathrm{SrTiO}_{3}$ phonon peaks. The arrows indicate the peaks originating from the $\mathrm{SrTiO}_{3}$ phonon. All the spectra are normalized to the Si peaks at $\sim 960 \mathrm{~cm}^{-1}$. 


\section{ASSOCIATED CONTENT}

This material is available free of charge via the Internet at http://pubs.acs.org.

Experimental procedures and a lattice mismatch calculation of (100)- and (111)-oriented $\mathrm{SrTiO}_{3}$ on graphene substrate and temperature-dependent sheet resistance of $\mathrm{SrTiO}_{3}$ thin films (PDF)

\section{AUTHOR INFORMATION}

\section{Corresponding Author}

* E-mail: kimsungwng@skku.edu (S.W.K.)

* E-mail: choiws@skku.edu (W.S.C.)

\section{Author Contributions}

${ }^{\perp}$ These authors contributed equally to this work.

\section{Notes}

The authors declare no competing financial interest.

\section{ACKNOWLEDGMENT}

This work was supported by Basic Science Research Programs through the National Research Foundation of Korea (NRF) (NRF-2014R1A2A2A01006478 (W.S.C.) and NRF2013R1A1A2057523 (S.A L.)). This work was also supported by IBS-R011-D1 (J.-Y.H., E.S.K., S.W.K., and W.S.C.). 


\section{REFERENCES}

(1) Hwang, H. Y.; Iwasa, Y.; Kawasaki, M.; Keimer, B.; Nagaosa, N.; Tokura, Y., Emergent Phenomena at Oxide Interfaces. Nat. Mater. 2012, 11, 103-113.

(2) Zubko, P.; Gariglio, S.; Gabay, M.; Ghosez, P.; Triscone, J.-M., Interface Physics in Complex Oxide Heterostructures. Annu. Rev. Condens. Matter Phys. 2011, 2, 141-165.

(3) Wang, J.; Neaton, J. B.; Zheng, H.; Nagarajan, V.; Ogale, S. B.; Liu, B.; Viehland, D.; Vaithyanathan, V.; Schlom, D. G.; Waghmare, U. V.; Spaldin, N. A.; Rabe, K. M.; Wuttig, M.; Ramesh, R., Epitaxial $\mathrm{BiFeO}_{3}$ Multiferroic Thin Film Heterostructures. Science 2003, 299, 1719-1722.

(4) Suntivich, J.; May, K. J.; Gasteiger, H. A.; Goodenough, J. B.; Shao-Horn, Y., A Perovskite Oxide Optimized for Oxygen Evolution Catalysis from Molecular Orbital Principles. Science 2011, 334, 1383-1385.

(5) Ohta, H.; Kim, S.; Mune, Y.; Mizoguchi, T.; Nomura, K.; Ohta, S.; Nomura, T.; Nakanishi, Y.; Ikuhara, Y.; Hirano, M.; Hosono, H.; Koumoto, K., Giant Thermoelectric Seebeck Coefficient of a Two-Dimensional Electron Gas in $\mathrm{SrTiO}_{3}$. Nat. Mater. 2007, 6, 129-134.

(6) McKee, R. A.; Walker, F. J.; Chisholm, M. F., Crystalline Oxides on Silicon: The First Five Monolayers. Phys. Rev. Lett. 1998, 81, 3014-3017.

(7) Choi, M.; Posadas, A.; Dargis, R.; Shih, C.-K.; Demkov, A. A.; Triyoso, D. H.; David Theodore, N.; Dubourdieu, C.; Bruley, J.; Jordan-Sweet, J., Strain Relaxation in Single Crystal $\mathrm{SrTiO}_{3}$ Grown on Si (001) by Molecular Beam Epitaxy. J. Appl. Phys. 2012, 111, 064112.

(8) Niu, G.; Penuelas, J.; Largeau, L.; Vilquin, B.; Maurice, J. L.; Botella, C.; Hollinger, G.; Saint-Girons, G., Evidence for the Formation of Two Phases During the Growth of $\mathrm{SrTiO}_{3}$ on Silicon. Phys. Rev. B 2011, 83, 054105. 
(9) Kolpak, A. M.; Walker, F. J.; Reiner, J. W.; Segal, Y.; Su, D.; Sawicki, M. S.;

Broadbridge, C. C.; Zhang, Z.; Zhu, Y.; Ahn, C. H.; Ismail-Beigi, S., Interface-Induced Polarization and Inhibition of Ferroelectricity in Epitaxial $\mathrm{SrTiO}_{3} / \mathrm{Si}$. Phys. Rev. Lett. 2010, $105,217601$.

(10) Warusawithana, M. P.; Cen, C.; Sleasman, C. R.; Woicik, J. C.; Li, Y.; Kourkoutis, L. F.; Klug, J. A.; Li, H.; Ryan, P.; Wang, L.-P.; Bedzyk, M.; Muller, D. A.; Chen, L.-Q.; Levy, J.; Schlom, D. G., A Ferroelectric Oxide Made Directly on Silicon. Science 2009, 324, $367-$ 370.

(11) Reiner, J. W.; Kolpak, A. M.; Segal, Y.; Garrity, K. F.; Ismail-Beigi, S.; Ahn, C. H.; Walker, F. J., Crystalline Oxides on Silicon. Adv. Mater. 2010, 22, 2919-2938.

(12) Niu, G.; Saint-Girons, G.; Vilquin, B.; Delhaye, G.; Maurice, J.-L.; Botella, C.; Robach, Y.; Hollinger, G., Molecular Beam Epitaxy of $\mathrm{SrTiO}_{3}$ on $\mathrm{Si}(001)$ : Early Stages of the Growth and Strain Relaxation. Appl. Phys. Lett. 2009, 95, 062902.

(13) Forst, C. J.; Ashman, C. R.; Schwarz, K.; Blochl, P. E., The Interface between Silicon and a High-k Oxide. Nature 2004, 427, 53-56.

(14) Hu, X.; Li, H.; Liang, Y.; Wei, Y.; Yu, Z.; Marshall, D.; Edwards, J.; Droopad, R.; Zhang, X.; Demkov, A. A.; Moore, K.; Kulik, J., The Interface of Epitaxial $\mathrm{SrTiO}_{3}$ on Silicon: In Situ and Ex Situ Studies. Appl. Phys. Lett. 2003, 82, 203-205.

(15) Goncharova, L. V.; Starodub, D. G.; Garfunkel, E.; Gustafsson, T.; Vaithyanathan, V.; Lettieri, J.; Schlom, D. G., Interface Structure and Thermal Stability of Epitaxial $\mathrm{SrTiO}_{3}$ Thin Films on Si (001). J. Appl. Phys. 2006, 100, 014912.

(16) Li, H.; Hu, X.; Wei, Y.; Yu, Z.; Zhang, X.; Droopad, R.; Demkov, A. A.; Edwards, J.; Moore, K.; Ooms, W.; Kulik, J.; Fejes, P., Two-Dimensional Growth of High-Quality Strontium Titanate Thin Films on Si. J. Appl. Phys. 2003, 93, 4521-4525. 
Alaskar, Y.; Arafin, S.; Wickramaratne, D.; Zurbuchen, M. A.; He, L.; McKay, J.;

Lin, Q.; Goorsky, M. S.; Lake, R. K.; Wang, K. L., Towards van der Waals Epitaxial Growth of GaAs on Si using a Graphene Buffer Layer. Adv. Funct. Mater. 2014, 24, 6629-6638.

Shi, Y.; Zhou, W.; Lu, A.-Y.; Fang, W.; Lee, Y.-H.; Hsu, A. L.; Kim, S. M.; Kim, K. K.; Yang, H. Y.; Li, L.-J.; Idrobo, J.-C.; Kong, J., van der Waals Epitaxy of MoS 2 Layers Using Graphene As Growth Templates. Nano Lett. 2012, 12, 2784-2791.

(19) Hong, S.; Kim, E. S.; Kim, W.; Jeon, S.-J.; Lim, S. C.; Kim, K. H.; Lee, H.-J.; Hyun, S.; Kim, D.; Choi, J.-Y.; Lee, Y. H.; Baik, S., A Hybridized Graphene Carrier Highway for Enhanced Thermoelectric Power Generation. Phys. Chem. Chem. Phys. 2012, 14, 1352713531.

(20) Shibata, T.; Takano, H.; Ebina, Y.; Kim, D. S.; Ozawa, T. C.; Akatsuka, K.; Ohnishi, T.; Takada, K.; Kogure, T.; Sasaki, T., Versatile van der Waals Epitaxy-Like Growth of Crystal Films using Two-Dimensional Nanosheets as a Seed Layer: Orientation Tuning of $\mathrm{SrTiO}_{3}$ Films along Three Important Axes on Glass Substrates. J. Mater. Chem. C 2014, 2, 441-449.

(21) Woo, S.; Jeong, H.; Lee, S. A.; Seo, H.; Lacotte, M.; David, A.; Kim, H. Y.; Prellier, W.; Kim, Y.; Choi, W. S., Surface Properties of Atomically Flat Poly-Crystalline $\mathrm{SrTiO}_{3}$. Sci. Rep. 2015, 5, 8822 .

(22) Nakada, K.; Ishii, A., Migration of Adatom Adsorption on Graphene using DFT Calculation. Solid State Commun. 2011, 151, 13-16.

(23) Lee, S. A.; Jeong, H.; Woo, S.; Hwang, J.-Y.; Choi, S.-Y.; Kim, S.-D.; Choi, M.; Roh, S.; Yu, H.; Hwang, J.; Kim, S. W.; Choi, W. S., Phase Transitions via Selective Elemental Vacancy Engineering in Complex Oxide Thin Films. Sci. Rep. 2016, 6, 23649.

(24) Niu, F.; Wessels, B. W., Surface and Interfacial Structure of Epitaxial $\mathrm{SrTiO}_{3} \mathrm{Thin}$ Films on (0 0 1) Si Grown by Molecular Beam Epitaxy. J. Cryst. Growth 2007, 300, 509-518. 
Choi, W. S.; Rouleau, C. M.; Seo, S. S. A.; Luo, Z.; Zhou, H.; Fister, T. T.; Eastman, J. A.; Fuoss, P. H.; Fong, D. D.; Tischler, J. Z.; Eres, G.; Chisholm, M. F.; Lee, H. N., Atomic Layer Engineering of Perovskite Oxides for Chemically Sharp Heterointerfaces. Adv. Mater. 2012, 24, 6423-6428.

(26) Yu, Z.; Ramdani, J.; Curless, J. A.; Overgaard, C. D.; Finder, J. M.; Droopad, R.; Eisenbeiser, K. W.; Hallmark, J. A.; Ooms, W. J.; Kaushik, V. S., Epitaxial Oxide Thin Films on Si (001). J. Vac. Sci. Technol., B 2000, 18, 2139-2145.

Wei, Y.; Hu, X.; Liang, Y.; Jordan, D. C.; Craigo, B.; Droopad, R.; Yu, Z.; Demkov, A.; Edwards, J. L.; Ooms, W. J., Mechanism of Cleaning Si (100) Surface using Sr or SrO for the Growth of Crystalline $\mathrm{SrTiO}_{3}$ Films. J. Vac. Sci. Technol., B 2002, 20, 1402-1405.

(28) Liu, L.; Ryu, S.; Tomasik, M. R.; Stolyarova, E.; Jung, N.; Hybertsen, M. S.;

Steigerwald, M. L.; Brus, L. E.; Flynn, G. W., Graphene Oxidation: Thickness-Dependent Etching and Strong Chemical Doping. Nano Lett. 2008, 8, 1965-1970.

(29) Tenne, D. A.; Farrar, A. K.; Brooks, C. M.; Heeg, T.; Schubert, J.; Jang, H. W.; Bark, C. W.; Folkman, C. M.; Eom, C. B.; Schlom, D. G., Ferroelectricity in Nonstoichiometric $\mathrm{SrTiO}_{3}$ Films Studied by Ultraviolet Raman Spectroscopy. Appl. Phys. Lett. 2010, 97, 142901. (30) Hilt Tisinger, L.; Liu, R.; Kulik, J.; Zhang, X.; Ramdani, J.; Demkov, A. A., Ultraviolet-Raman Studies of $\mathrm{SrTiO}_{3}$ Ultrathin Films on Si. J. Vac. Sci. Technol., B 2003, 21, $53-56$. 


\section{Supporting Information}

\section{Highly oriented $\mathrm{SrTiO}_{3}$ thin film on graphene}

\section{substrate}

Sang A Lee ${ }^{\dagger, \ddagger, \perp}$, Jae-Yeol Hwang ${ }^{\S, \perp}$, Eun Sung Kim ${ }^{\S}$, Sung Wng Kim ${ }^{*}, \|$, and Woo Seok

$\mathrm{Choi}^{*}+\dagger$

${ }^{\dagger}$ Department of Physics, Sungkyunkwan University, Suwon, 16419, Korea

${ }^{\ddagger}$ Institute of Basic Science, Sungkyunkwan University, Suwon, 16419, Korea

${ }^{\S}$ Center for Integrated Nanostructure Physics, Institute for Basic Science (IBS), Suwon 16419,

Korea

" Department of Energy Sciences, Sungkyunkwan University, Suwon 16419, Korea

* E-mail: kimsungwng@skku.edu (S.W.K.)

* E-mail: choiws@skku.edu (W.S.C.)

${ }^{\perp}$ These authors contributed equally to this work. 


\section{EXPERIMENTAL PROCEDURES}

\section{Thin film growth and structural characterization.}

\section{1a. Graphene growth.}

Graphene was synthesized on a chemical-mechanical polished (CMP) $\mathrm{Cu}$ foil (70 $\mu \mathrm{m}$ thickness, Nilaco Co., Tokyo, Japan) in a 3-in quartz tube through the thermal chemical vapor deposition (CVD) method. First, the $\mathrm{Cu}$ foil was heated up to $1060^{\circ} \mathrm{C}$ for $40 \mathrm{~min}$ and annealed for 90 min under $\mathrm{H}_{2}$ atmosphere. After the annealing process, $\mathrm{H}_{2}$ and $\mathrm{CH}_{4}$ gases were injected into the furnace to synthesize graphene at $1060^{\circ} \mathrm{C}$ for $5 \mathrm{~min}$. Finally, the $\mathrm{Cu}$ foil was rapidly cooled down to room temperature without any gas flow. Then, the few-layergraphene film on the $\mathrm{Cu}$ foil was transferred onto an $a-\mathrm{SiO}_{2}$ (amorphous phase with $300 \mathrm{~nm}$ thickness)/Si substrate by successive wet etching processes, including coating of poly(methyl methacrylate) (PMMA; MicroChem, e-beam resist, 950 k C4), etching (Transene, Danvers, MA, CE-100) of residual $\mathrm{Cu}$, and removal of PMMA using acetone. The sample size of graphene on the $a-\mathrm{SiO}_{2} / \mathrm{Si}$ substrate was $\sim 10 \times 10 \mathrm{~mm}^{2}$.

\section{1b. $\mathrm{SrTiO}_{3}$ growth.}

$\mathrm{SrTiO}_{3}$ thin films were grown on $a-\mathrm{SiO}_{2} / \mathrm{Si}$ single-crystalline substrates with and without a graphene by using pulsed laser deposition (PLD) at $700^{\circ} \mathrm{C}$. A laser $(248 \mathrm{~nm}$; Lightmachinery, IPEX 864) fluence of $2 \mathrm{~J} / \mathrm{cm}^{2}$ and repetition rate of $3 \mathrm{~Hz}$ were used. The oxygen partial pressure was $10^{-6}$ Torr. Low oxygen partial pressure was employed in order to prevent oxidization of the graphene layer. We found that higher temperature or oxygen partial pressure resulted in the formation of polycrystalline phase in the $\mathrm{SrTiO}_{3}$ thin films. Details of the chamber configuration can be found elsewhere. ${ }^{26}$ The atomic structure and the crystal orientations of the $\mathrm{SrTiO}_{3}$ thin films were characterized by high resolution x-ray diffraction 
(XRD). The thickness of the $\mathrm{SrTiO}_{3}$ thin film was determined through x-ray reflectometry (XRR) as being $\sim 40 \pm 1 \mathrm{~nm}$.

\section{Transmission electron microscopy (TEM).}

We prepared the sample foil by using the conventional method, which entailed mechanical thinning to $\sim 10 \mu \mathrm{m}$ and subsequent ion beam milling to electron transparency at an acceleration voltage of $0.5-3.5 \mathrm{kV}$ using an Ar ion beam. The atomic strcuture was observed using a TEM instrument (JEOL JEM-ARM200F, JEOL, Ltd., Japan). The probe diameter of the beam was $\sim 0.9 \AA$.

\section{Raman spectroscopy and resistivity measurements.}

The Raman spectra of all samples were acquired using a Renishaw Raman spectrometer (Renishaw inVia micro-Raman spectrometer) with an excitation wavelength of $532 \mathrm{~nm}$. All the spectra are normalized to the Si peaks at $\sim 960 \mathrm{~cm}^{-1}$. Resistivity was measured as a function of temperature $(\rho(T))$ by using a low-temperature closed-cycle refrigerator. The measurements were performed from $300 \mathrm{~K}$ to $20 \mathrm{~K}$ by using the Van der Pauw method with In electrodes and $\mathrm{Au}$ wires. 
(a)

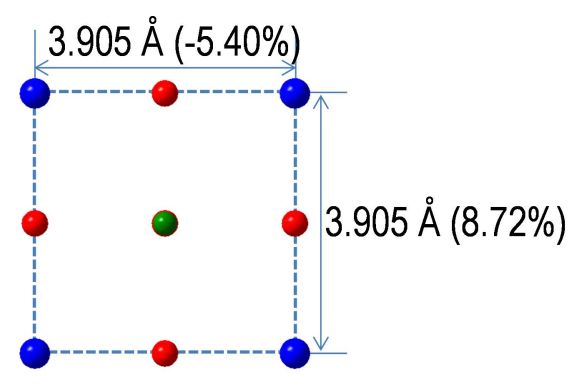

$3.705 \AA$

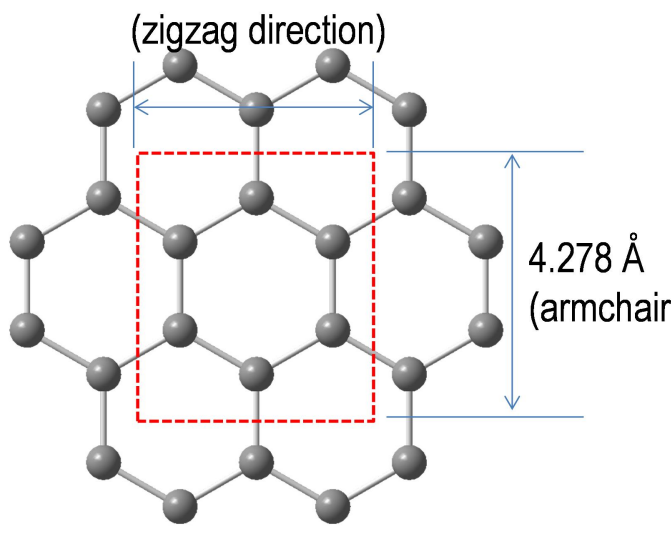

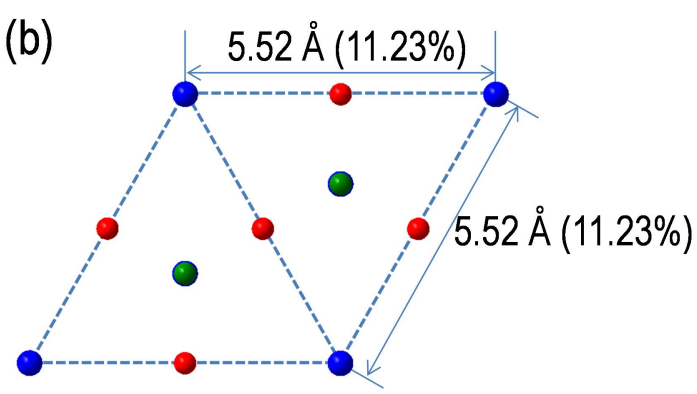

$4.94 \AA$

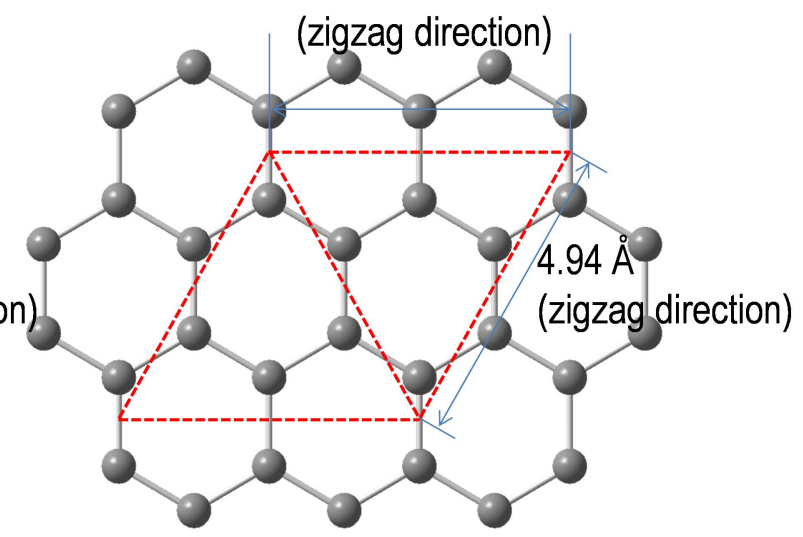

Figure S1. Simple lattice mismatch calculation of (a) (001)- and (b) (111)-oriented $\mathrm{SrTiO}_{3}$.

The epitaxial strain imposed by graphene on (001) $\mathrm{SrTiO}_{3}$ thin film is $\sim 8.72 \%$ (armchair direction) and $-5.40 \%$ (zigzag direction) considering simple rectangular box on top of hexagonal network. The lattice mismatch of (111) $\mathrm{SrTiO}_{3}$ thin film is $\sim 11.23 \%$ (zigzag direction). 


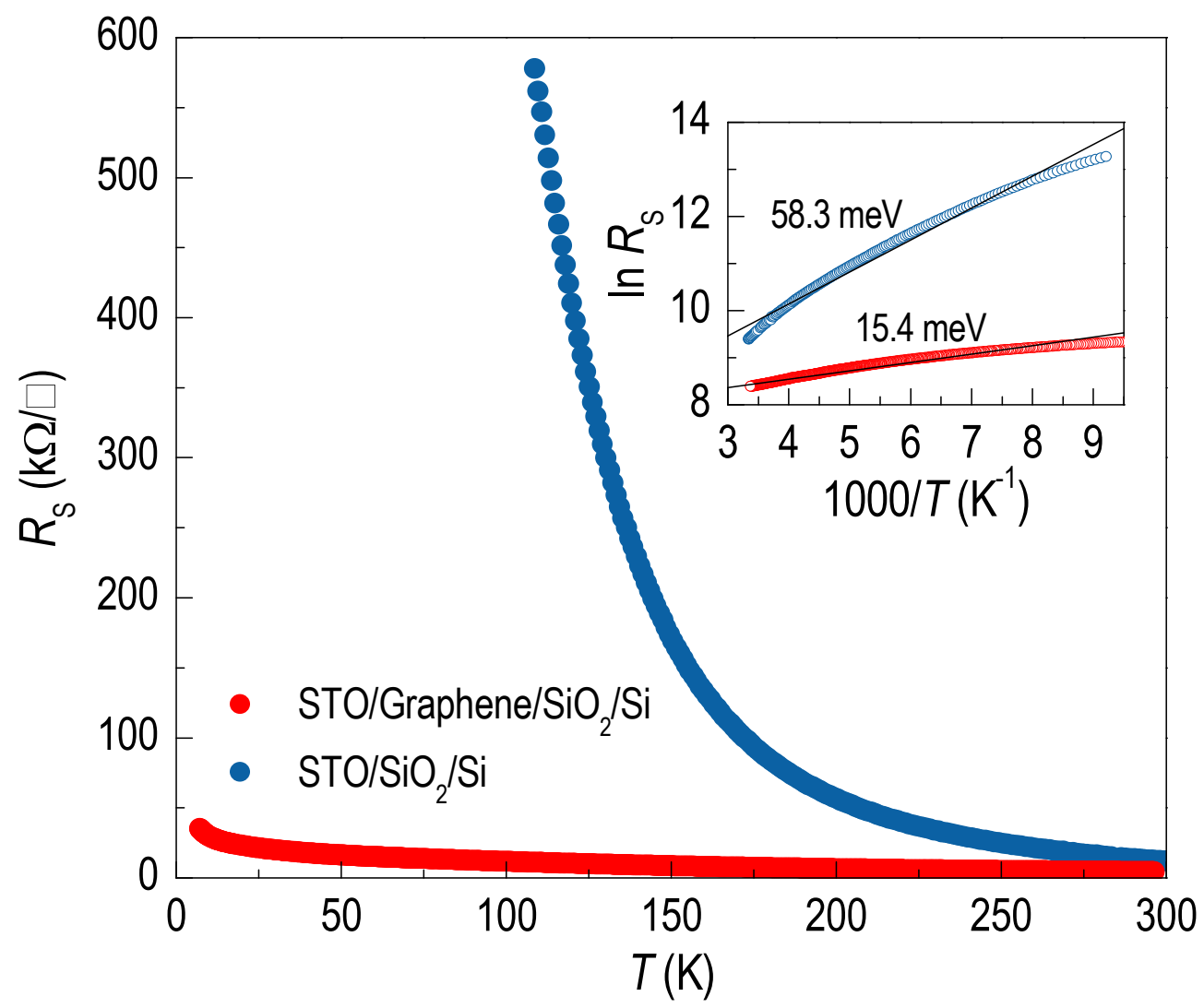

Figure S2. Temperature-dependent sheet resistance $\left(R_{\mathrm{s}}(T)\right)$ of $\mathrm{SrTiO}_{3}$ thin films with and without graphene. The inset shows the Arrhenius plot of the electric conduction through $\mathrm{SrTiO}_{3}$ with and without the graphene layer. The calculated activation energies are 58.3 and $15.4 \mathrm{meV}$ for $\mathrm{SrTiO}_{3}$ without and with graphene, respectively.

The graphene substrate induces lower resistance in the $\mathrm{SrTiO}_{3}$ thin film by establishing the crystal structure as well as enabling electric conduction. Figure S2 shows the sheet resistance of the thin films as a function of temperature. Since the $\mathrm{SrTiO}_{3}$ thin films were grown at low oxygen partial pressure, abundant oxygen vacancies could be induced, which resulted in the generation of free charge carriers. However, the resistivities reveal semiconducting temperature dependence, which might be due to the disorder induced by the grain boundaries. Nevertheless, the $\mathrm{SrTiO}_{3}$ thin film on graphene substrate showed lower electric resistance 
over the whole temperature range studied compared to that without graphene. The activation energy obtained from Arrhenius plot was also lower (from 58.3 to $15.4 \mathrm{meV}$ ), as shown in the inset of Figure S2. These behaviors might originate from further enhanced carrier scattering by atomic disorders in the amorphous $\mathrm{SrTiO}_{3}$ thin film. The presence of the intermediate layer in the $\mathrm{SrTiO}_{3}$ thin film without graphene could also be detrimental for the electric conduction. On the other hand, the weak bonding nature facilitating the partial epitaxy between $\mathrm{SrTiO}_{3}$ thin film and graphene substrate seems to be beneficial for the delocalized $3 d^{1}$ electrons in the $\mathrm{Ti}$ band of $\mathrm{SrTiO}_{3}$ to flow. 